See discussions, stats, and author profiles for this publication at: https://www.researchgate.net/publication/331257209

\title{
Impact of a Brief Worry Based Cognitive Therapy Group in Psychosis: A Study of Feasibility and Acceptability.
}

Article in The Cognitive Behaviour Therapist · February 2019

DOI: $10.1017 / / 1754470 \times 1900014 x$

\section{CITATIONS}

6 authors, including:

Lucy White

Solent NHS Trust

7 PUBLICATIONS O CITATIONS

SEE PROFILE

Jo Tedbury

Solent NHS Trust

5 PUBLICATIONS OCITATIONS

SEE PROFILE
READS

56

Thomas Richardson

Solent NHS Trust

110 PUBLICATIONS 1,655 CITATIONS

SEE PROFILE

Jane Butt

University of Southampton

3 PUBLICATIONS O CITATIONS

SEE PROFILE

Some of the authors of this publication are also working on these related projects:

Project $\quad$ Test of a Cognitive Model of Cannabis related Paranoia View project

Home environment: Association with hyperactivity/impulsivity in children with ADHD and their non-ADHD siblings View project 
This article has been accepted for publication in the Cognitive Behaviour Therapist and will appear in a revised form, subsequent to peer review and/or editorial input by Cambridge University Press, published by Cambridge University Press.

Copyright for the article belongs to Cambridge University Press. 
Impact of a Brief Worry Based Cognitive Therapy Group in Psychosis: A Study of Feasibility and Acceptability.

\begin{tabular}{|r|l|}
\hline Journal: & The Cognitive Behaviour Therapist \\
\hline Manuscript ID & CBT-00463-18.R1 \\
\hline Manuscript Type: & Original Research \\
\hline Keywords: & psychosis, CBT, worry, group psychotherapy, persecutory delusions \\
\hline
\end{tabular}

\section{SCHOLARONE ${ }^{\text {IM }}$ \\ Manuscripts}


WORRY GROUP FOR PSYCHOSIS

Original Research

\title{
Impact of a Brief Worry Based Cognitive Therapy Group in Psychosis: A Study of Feasibility and Acceptability.
}

\begin{abstract}
Background: Previous research suggests that CBT focusing on worry in those with persecutory delusions reduces paranoia, severity of delusions and associated distress.

Aims: This preliminary case series aimed to see whether it is feasible and acceptable to deliver worry focused CBT in a group setting to those with psychosis. A secondary aim was to examine possible clinical changes.

Methods: Two groups totalling 11 participants were run for 7 sessions using the WIT trial manual. Qualitative and quantitative data about the experience of being in the group was also collected via questionnaires as was data on number of sessions attended. Measures were delivered pre and post group and at 3 month follow up. These included a worry scale, a measure of delusional belief and associated distress and quality of life measures.

Results: Of the 11 participants who started the group, 9 completed the group. Qualitative and quantitative feedback indicated that most of the participants found it acceptable and helpful, and that discussing these issues in a group setting was not only tolerable but often beneficial. Reliable Change Index indicated that $6 / 7$ of the group members showed reliable reductions in their levels of worry post group and 5/7 at follow up. There positive changes on other measures which appeared to be more pronounced at follow-up.
\end{abstract}

Conclusions: Delivering a worry intervention in a group format appears to be acceptable and feasible. Further research with a larger sample and control group is indicated to test the clinical effectiveness of this intervention.

Keywords: Psychosis, paranoia, CBT, worry, group, persecutory delusions. 
WORRY GROUP FOR PSYCHOSIS

\section{Learning Objectives}

- Understand the role of worry in psychosis.

- Learn about possible feasibility of working on worry in a group setting.

- Be aware of potential clinical changes from the group..

- Consider acceptability for participants of working on worries in a group setting. 
WORRY GROUP FOR PSYCHOSIS

\section{Impact of a Brief Worry Based Cognitive Therapy Group in Psychosis: A Study of Feasibility and Acceptability.}

\section{Introduction}

Cognitive Behavioural Therapy (CBT) is recommended for psychosis as highlighted in the National Institute of Clinical Excellence guidelines (NICE, 2014). This usually takes the form of one-to-one sessions, the aims of which can be defined as "reducing distress, increasing confidence and reengaging in activity...initially the therapist and patient develop a shared understanding, with the focus on what is maintaining the current problems and what can immediately be changed...the clinician plays a collaborative role: drawing up a list of goals with the patient" (Freeman, 2013). Historically this intervention has used psychological models which inform and illustrate our understanding of the development of psychosis and the maintenance of its symptoms e.g. Morrison (2001). In more recent years research has begun to dismantle the umbrella term of 'psychosis' into specific problems: Freeman (2016) argues that experiences such as grandiosity, hearing voices and paranoia are different from one another, with different causes. Thus the focus has shifted to considering these as individual experiences rather than symptoms of psychosis, and as such more targeted models and interventions are beginning to be developed. . Of note is the work of Freeman (2016) who has researched the maintenance factors involved in persecutory delusions, one of which is worry.

Research has shown that the majority of those with persecutory delusions have levels of worry which are similar to those with GAD (Startup, Freeman \& Garety, 2007), and high worry predicts greater paranoia in both those with psychosis and the general population (Freeman et al., 2012; Startup, Freeman \& Garety, 2007). Worry is believed to be linked to persecutory delusions as it "brings implausible fearful ideas to mind, keeps them there, and exacerbates the distress" (Freeman, 2016, p.687). The relationship is also likely to work both 


\section{WORRY GROUP FOR PSYCHOSIS}

ways with greater paranoia also increasing levels of worry (Freeman, 2016). In the Worry Intervention Trial (WIT), Freeman et al. (2015) investigated whether using CBT to reduce worry would also reduce persecutory delusions in patients with psychotic disorders, They conducted a parallel, single-blind, randomised controlled trial. They randomly assigned 150 eligible participants to either a six session, 1-1 worry-reduction CBT intervention completed over 8 weeks added to standard care, or standard care alone. They concluded that long- standing delusions were significantly reduced by a brief intervention targeting worry.

Evidence from the Hearing Voices Groups movement suggests that there is value to participants in the shared experience of attending a group and being in a safe context in which to share experiences (Ruddle, Mason and Wykes 2011). In a recent feasibility study, Isham et al (2018) assigned thirteen participants to an 8 week worry group or control. They adapted the manual from the WIT trial (Freeman et al., 2015) for a group format, and concluded that the group was feasible and showed positive outcomes in worry, however there were no changes in paranoia and outcomes in delusional beliefs were not maintained at follow-up. They suggest that the effects are perhaps not as strong as in one-to-one-interventions. However it is important to note that this was a feasibility study and was not statistically powered to detect changes.

This current study aimed to build on the evidence of the effectiveness of targeting worry in paranoia from the WIT trial, and see whether it is feasible and acceptable for this to be delivered in a group format. A secondary aim is to look at potential impact on symptoms, functioning and quality of life..

\section{Method}

\section{Design}


WORRY GROUP FOR PSYCHOSIS

This study was a feasibility and acceptability study, as part of this a preliminary case series was used. NHS ethics approval was not needed as this was evaluating routine clinical practice, approval was granted by the trust service evaluation and audit team. Measures were completed as part of routine clinical practice for the group being evaluated and all participants gave written consent for their data to be used anonymously for this service evaluation and were deemed by their clinician to have capacity to give informed consent.

\section{Setting}

The group was delivered within a National Health Service (NHS) community mental health team (CMHT) for adults with severe and enduring mental health problems and the Early Intervention in Psychosis (EIP) team. The psychosis treatment pathway offers evidence based interventions in a 1-1 and family setting. The group was developed and facilitated by a CBT Therapist (L.W.) and Clinical Psychologist (H.C.) both of whom have specialist CBT training and experience in working with people with psychosis, including training on the delivery of a worry intervention for paranoia.

\section{Intervention}

A 7-week programme was developed following the components and structure reported in the WIT trial (e.g. understanding, overcoming and staying ahead of worry). The main core element of the original WIT manual which was not included was Emotional Processing and Metacognitive Awareness as it was not included in the pilot by Isham et al (2018) and was felt by the current facilitators that it would take too long and not work well in a group format. The WIT manual was given to each group member. Each session lasted two hours, with a short break half way through. It followed the following format: homework, check -in from last week, main topic of the session, homework setting and considering barriers to practise/homework. A brief telephone call was made to each participant ever week between group sessions to check in with homework and address any arising issues. 


\section{WORRY GROUP FOR PSYCHOSIS}

\section{Procedure}

Participants were referred by the CMHT and EIP teams. The inclusion criteria were: a primary diagnosis of psychosis with evidence of persecutory beliefs and significant worry. Specific examples of worry and the impact on function were given in the assessment; however no standardised measure was used for screening.

Exclusion criteria were: a primary diagnosis of personality disorder, current alcohol or substance dependency. Each suitable participant was seen by one of the facilitators for a pregroup assessment to discuss practicalities and assess their ability to attend and participate in the group. Eligible participants met one of the researchers who were separate from the facilitators to complete consent forms and pre-group, post and follow-up questionnaires.

Participants were asked to complete an evaluation feedback form at the end of the group. This was anonymous: participants did not have to put their name on and they gave the form back to a researcher who did not facilitate the group. This consisted of two likert scales to rate how helpful they found the worry group and how easy it was to follow what was being discussed (see Appendix 1). Free text boxes were presented for the following questions:

- What was the most helpful part about the group?

- What could we improve about the group?

- How did you find talking about worry and paranoia in a group with other people?

- Is there anything else you want to say?

Participants were offered $£ 10$ per time point to cover their expenses. The following data is from the first two of these groups which were run.

\section{Measures}


WORRY GROUP FOR PSYCHOSIS

The following measures were administered pre, post and at 3- month follow up. (Cronbach's alpha for current sample pre group given below).

Psychotic-Symptoms Rating Scale (PSYRATS) - delusion scale. (Haddock, McCarron, Tarrier, Fargher and Tarrier, 1999): a 6-item clinician-rated measure of severity of delusional beliefs via categories such as 'Disruption to life caused by beliefs'; $\alpha=.74$.

Penn State Worry Questionnaire (PSWQ) (Meye, Miller, Metzger, and Borkovec, 1990): a 16-item self-report measure of intensity, duration and distress associated with worry such as 'My worries overwhelm me'; $\alpha=.90$.

Green et al paranoid thoughts scale (GPTS) (Green, Freeman, Kuipers and Bebbington, 2008): A 32-item self-report measure of two dimensions of paranoid thinking; social reference and social persecution. An example item is 'People have been checking up on me'; $\alpha=.95$

Choice of outcome in CBT for psychoses - short form (CHOICE) (Greenwood, Sweeney, Williams, Garety, Kuipers and Scott, 2010): A 11-item self-report measure developed to ask people what they want to address in CBT for psychosis. For example 'Positive ways of relating to people'; $\alpha=.92$.

Work and Social Adjustment Scale (WSAS) (Mund, Marks, Shear and Greist, 2002): A 5-item self-report asking people how their problems affect their daily functioning such as work and leisure etc. Example questions include 'Because of my problem my home management is impaired'; $\alpha=.57$.

The Recovering Quality of Life (REQOL)- 10 item version. (Keetharuth et al, 2018): this self-report measure assesses the quality of life for people with a range of mental health problems. Questions include 'I felt hopeful about my future'; $\alpha=.89$

\section{Participant characteristics}




\section{WORRY GROUP FOR PSYCHOSIS}

Figure 1 displays a flow diagram of the recruitment of participants into the study. Fourteen people were initially screened for the group of whom eleven started the group and completed pre questionnaires. Of these, eight then completed the group and post measures and six completed follow-up measures. Two of the participants (both of whom completed the group) were also undergoing one-to-one CBT for psychosis at the same time as attending the group. A further one completer started the assessment/formulation stage of therapy in between post-group and follow-up measures being completed.

\section{***Insert Figure 1 here**}

Of the 11 participants who took part in the group and service evaluation, two participants were under the EIP service. The group was $73 \%(n=8)$ female, ages ranged from 17 to 60 years, with a mean age of 46 years. The sample was $90 \%$ White Ethnicity $(n=10)$ and $9.1 \%$ $(n=1)$ Asian ethnicity. In terms of diagnosis, four had a diagnosis of schizophrenia, two psychotic depression, two first episode psychosis, one delusional disorder, one schizoaffective disorder and one with a current diagnosis of post psychotic personality changes and a past diagnosis of schizophrenia. Three of the 11 participants had co-morbid diagnosis of personality disorder traits or substance use. Pre scores on the PSWQ ranged from 41 to 77 out of $80,5 / 8$ participants scored above the cut-off of 65 recommended for detecting Generalized Anxiety Disorder (GAD) (Douglas, Mennin, Heimberg \& Turk, 2003).

\section{Statistical Analysis}

The only missing data was for the CHOICE where two questions were missing for one individual each. For these the mode response for that measure for that client was used and the total scale score was calculated as normal. The sample size was too small for statistics such as t-tests or a Multiple analysis of Variance to detect changes in measures from pre therapy to post therapy. The Reliable Change Index (RCI) (Jacobson \& Truax, 1991) is useful with small sample sizes as it looks at individual changes in scores (Zahra \& Hedge, 
WORRY GROUP FOR PSYCHOSIS

2010). The RCI is calculated by dividing the changes in scores by the standardised difference (in this case calculated using the standard deviation and chronbachs alpha in the current sample pre group), this then gives a change score needed to reach corresponding to a standardised z-score of less than -1.96 or above 1.96 of which would less than $5 \%$ likely to occur by chance and therefore likely represents a reliable change rather than error (de Souza Costa \& De Paula, 2015). An online calculator was used for the current study (https://www.psychoutcomes.org/OutcomesMeasurement/ReliableChangeIndex).

\section{Results}

\section{Group Attendance}

Of the 11 participants who took part in the service evaluation and started the group, 2 dropped out of the group. One of these attended two of the first three sessions and then no further sessions. The group facilitators reported that they were highly anxious in the first session and said they felt daunted by the homework and being called in between sessions. In another session they appeared distracted, and they then went abroad for several weeks. The second participant who dropped out attended the first two session and then no further sessions. The facilitators reported they were highly anxious in the first session and were not able to take in the content, they also reported being physically ill one week.

For the nine participants who completed the group, two attended all 7 sessions, five attended 6/7 sessions, one attended 5/7 sessions and one participant attended 4/7 sessions. Thus the mean number of sessions attended for completers was 5.1/7.

\section{Participant Feedback}

Participants were asked to complete a feedback questionnaire, figure 3 shows that most participants rated the group as easy to follow and helpful. 


\section{WORRY GROUP FOR PSYCHOSIS}

**Insert Figure 3 here**

Table 1 displays qualitative feedback from 9 participants about what most helpful about the group, what could be done to improve the group, how they found talking about worry and paranoia with other people in a group and any other comments. The comments were largely positive with most saying that learning skills to reduce worry and learning from other people's experiences was helpful.

**Insert Table 1 here**

\section{Facilitator Feedback on Running the Group}

Informal feedback from the group facilitators was that worry periods were established and worries were able to be postponed for all participants who completed the group. This was done in week 3 (introducing worry periods) and then followed up in week 4 (boosting worry periods). The group used problem solving discussions to overcome barriers to the worry periods. Between-session phone calls were received well by participants and the facilitators believe they helped keep participants on track and helped with therapeutic relationship and therefore engagement, they were also used to help check in about worry periods. The facilitators also felt that normalising the universality of experiences was important, in line with the participant feedback.

\section{Reliable Change in Scores}

Figure 2 displays the results for the reliable change index, these compare changes in scores from pre group to post group and pre group to follow-up. This shows there was a reliable change in worry scores (PSWQ) for the majority of completers and just over half of those who started the group and this was largely maintained at follow-up. Severity of 
WORRY GROUP FOR PSYCHOSIS

delusional beliefs measured by PSYRATS reduced for only one individual by the end of the group, however the more had improved at follow up. Overall scores on the Green Paranoia Scale reveal improving gains from end of group to three month follow up. On the social persecution cognitions scale two individuals initially deteriorated before an improvement at follow-up. In terms of quality of life and daily functioning, there were reliable improvements as measured by the CHOICE, REQOL and WSAS at both post treatment and follow-up.

**Insert Figure 2 here**

\section{Discussion}

This study aimed to see whether a worry intervention for paranoia is feasible and acceptable when delivered in a group format, a secondary objective was a preliminary test of potential clinical changes via a case series The findings support the evidence generated by Freeman et al (2015) in their large scale RCT using the worry intervention in individual therapy, and tentatively suggest that this intervention is feasible to run in a group setting as evidenced by informal facilitator feedback, positive participant feedback and a relatively low dropout rate.

There was reliable change in worry scores for the majority of participants and this was largely maintained at follow up, in line with the findings of the group intervention of Isham et al (2018). Severity of delusional beliefs reduced for only one individual at post-treatment however the majority improved at follow up. In contrast, Isham et al (2018) found that delusional beliefs changes were not maintained at follow-up. This difference may be due to the control group in the Isham et al. study compared to a case series here. However it is important to note that due to the design and small sample size, as well as some participants having one-to-one therapy at the same time, the clinical effectiveness of the group cannot be determined from the current study. 


\section{WORRY GROUP FOR PSYCHOSIS}

This trend for maintaining or improving on gains from end of group to three month follow up is echoed in the Green Paranoia scale where reliable change increased to follow up, and indeed for persecution cognitions there was an initial deteriorating for two individuals before an improvement at follow up. This indicates that the gains from the group are perhaps on-going and that processing and consolidation of skills learnt continues after the group has finished. It might also be that the nature of the group is essentially exposure which for some might lead to an initial increase of paranoid cognitions before they start to reduce. There was also reliable change for the CHOICE and REQOL post-treatment follow-up suggesting that reduction in worry may have led to a wider improvement in quality of life for some. The WSAS also showed improvements suggesting a positive impact on functioning, however the internal reliability of this measure appeared to be low in the current sample so the results should be interpreted with caution. Also as previously stated the clinical effectiveness is uncertain in the current study.

The drop-out rate was $18 \%(n=2)$ is slightly higher than that reported by Freeman et al. (2015) of 9/73 for individual therapy (12\%) The current number of sessions attended of $5 / 7$ is comparable to Isham et al (2018) who reported an average of 5.7 out of 8 sessions However a larger sample size is required fully ascertain levels of drop-out.

Offering the intervention in a group format was considered to be the most helpful aspects of the group by a many participants in terms of reducing isolation, learning from others and sharing experiences with others. Four out of the nine also identified that a greater number of participants would have improved the group. The feedback indicated that although several were initially anxious about talking about worry and paranoia in the group, this was not sustained and they became comfortable in the group environment. In fact seven out of the nine respondents did not report any difficulty with this beyond the first session. This may seem counterintuitive given the nature of the client group however it suggests the group 
WORRY GROUP FOR PSYCHOSIS

environment might be more than just a non-specific effect: it might act as exposure, and a valuable opportunity for members to test out their fears and predictions about being around others.

It is important to note that one participant reported not understanding why the facilitators thought a group would help with paranoia. The group format may have been a factor for those who did not initially engage but there is no data available for this. It is also worth considering that though some participants asked for a larger group at the end of the seven sessions, this may have increased anxiety in the first session and led to higher levels of initial disengagement: the two participants who dropped out appeared to be highly anxious in the first couple of sessions. Thus a group setting may be challenging for some and lead to dropout. Being able to identify those who are likely to find the group setting hard and help prepare them may improve engagement.

This study is limited by a small sample size and no control group, with mostly white ethnicity. There was also no standardised measure of worry used for screening and thus what constituted a significant worry might have been somewhat subjective and open to the clinicians' interpretation, however scores on the PSQ were relatively high with many scoring above the cut-off for GAD. The feedback may be impacted by being paid for participation; however this feedback was collected independently and anonymously of the group facilitators. Two participants were also undergoing individual CBT whilst attending the group, so the potential clinical improvements for these individuals cannot be attributed solely to the group.

There is also the possibility of non-specific factors such as the effect of peer support here. Furture research could compare the effectiveness of a group to an individual worry intervention, and compare individual worry work to group worry work to a non-CBT group intervention to determine the relative contribution of non-specific factors such as social 


\section{WORRY GROUP FOR PSYCHOSIS}

support to outcomes. A randomised controlled trial is needed to fully assess clinical effectiveness compare outcomes to treatment as usual. It is also possible that those who did not finish the group or were lost at follow-up might not have benefited so much from the group, thus in future a intent to treat analysis would be necessary. However this preliminary case series has indicated that delivering worry based CBT for psychosis as a group is both feasible and acceptable. This may be useful in services as part of a 'stepped care' pathway for those with psychosis or as a preliminary intervention for those waiting for one-to-one CBT. 


\section{WORRY GROUP FOR PSYCHOSIS}

\section{Main Points}

- $\quad$ B B targeting worry may reduce distress in psychosis. Previous research has suggested this may be feasible in a group format.

- The current case series examined feasibility and acceptability and outcomes for 11 participants who attended a worry group.

- Dropout rates were acceptable (2/11).

- Qualitative feedback suggested that the group was helpful and that discussing worry about others in a group was beneficial.

- The majority of those who completed the group, and just over half of those who started the group showed reliable improvements in worry which were maintained in follow-up.

- There appeared to be improvements in distress and quality of life.

\section{Further Reading}

Freeman, D. (2016). Persecutory delusions: a cognitive perctive on understanding and treatment. The Lancet Psychiatry, 3(7), 685-692.

Freeman, D., Dunn, G., Startup, H., Pugh, K., Cordwell, J., Mander, H., Cernis, E., Wingham, G., Shirvell, K. and Kingdon, D. (2015). Effects of cognitive behaviour therapy for worry on persecutory delusions in patients with psychosis (WIT): a parallel, single-blind, randomised controlled trial with a mediation analysis. Lancet Psychiatry, 2, 305-13.

Isham, L., Grafahrend, H., Nickless, A., Pugh, K., Pleasants, S., Smedley, N., Freeman, D., \& Mulligan, A. (2018). Group-Based Worry Intevention for Persecutory Delusions: an Initial Feasibility Study. Behavioural and Cognitive Psychotherapy, 46, $619-625$. 


\section{WORRY GROUP FOR PSYCHOSIS}

\section{References}

de Souza Costa, D. \& De Paula, J. J. (2015). Usefulness of the reliable change index for psychology and psychiatry in clinical practice: A case report of cognitive-behavioral therapy. Clinical Neuropsychiatry, 12(5), 135-138.

Freeman, D. (2016). Persecutory delusions: a cognitive perctive on understanding and treatment. The Lancet Psychiatry, 3(7), 685-692.

Freeman, D., Dunn, G., Startup, H., Pugh, K., Cordwell, J., Mander, H., Cernis, E., Wingham, G., Shirvell, K. and Kingdon, D. (2015). Effects of cognitive behaviour therapy for worry on persecutory delusions in patients with psychosis (WIT): a parallel, single-blind, randomised controlled trial with a mediation analysis. Lancet Psychiatry, 2, 305-13.

Freeman, D. (2013). Cognitive-behavioral therapy for psychotic disorders. Psychiatric Times, 30, 16.

Freeman, D., Stahl, D., McManus, S., Meltzer, H., Brugha, T., Wiles, N., \& Bebbington, P. (2012). Insomnia, worry, anxiety and depression as predictors of the occurrence and persistence of paranoid thinking. Social psychiatry and psychiatric epidemiology, 47, 1195-1203.

Fresco, D. M., Mennin, D. S., Heimberg, R. G., \& Turk, C. L. (2003). Using the Penn State Worry Questionnaire to identify individuals with generalized anxiety disorder: A receiver operating characteristic analysis. Journal of behavior therapy and experimental psychiatry, 34, 283-291.

Green, C. E. L., Freeman, D., Kuipers, E. \& Bebbington, P. (2008). Measuring ideas of persecution and social reference: the Green et al. Paranoid Thought Scales (GPTS). Psychological Medicine, 38(1), 101-111. 
Greenwood, K. E, Sweeney, A., Williams, S., Garety, P., Kuipers, E., Scott, J. \& Peters, E. CHoice of Outcome In Cbt fr psychosEs (CHOICE) (2010). The Development of a New Service User-Led Outcome Measure of CBT for Psychosis. Schizophrenia Bulletin, 36(1), 126-135.

Haddock, G., McCarron, J., Tarrier, N., Faragher, E. and Tarrier, N. (1999). Scales to measure dimensions of hallucinations and delusions: the psychotic symptom rating scales (PSYRATS). Psychological Medicine, 29(4), 879-89.

Isham, L., Grafahrend, H., Nickless, A., Pugh, K., Pleasants, S., smedley, N., Freeman, D., \& Mulligan, A. (2018). Group-Based Worry Intevention for Persecutory Delusions: an Initial Feasibility Study. Behavioural and Cognitive Psychotherapy, 46, 619-625.

Jacobson, N. S. \& Truax, P. (1991). Clinical significance: a statistical approach to defining meaningful change in psychotherapy research. Journal of consulting and clinical psychology, 59(1), 12-19.Keetharuth, D., Brazier, J., Connell, J., Bjorner, J., Carlton, J., Taylor Buch, E., Rickets, T., McKendrick, K., Browne, J., Croudace, T. \& Barkham, M. (2018). Recovering Quality of Life (ReQoL): a new generic selfreported outcome measure for use with people experiencing mental health difficulties. British Journal of Psychiatry, 212(1), 42-49.

Meyer, T. J., Miller, M. L, and Metzger, R. L. and Borkovec, T. D. (1990). Development and validation of the penn state worry questionnaire. Behaviour Research and Therapy, 28(6), 487-496.

Morrison, A.P. (2001) The interpretation of intrusions in psychosis: An integrative cognitive approach to hallucinations and delusions. Behavioural and Cognitive Psychotherapy, 29(3), 257-276. 


\section{WORRY GROUP FOR PSYCHOSIS}

Mundt, J. C., Marks, I. M., Shear, M. K., Greist, J. M. (2002). The Work and Social Adjustment Scale: a simple measure of impairment in functioning. British Journal of Psychiatry, 180(5), 461-464.

National Institute for Health and Care Excellence (2014) Psychosis and schizophrenia in adults: prevention and management. NICE Guideline (CG178).

Ruddle, A., Mason, O. and Wykes, T. (2011). A review of hearing voices groups: Evidence and mechanisms of change. Clinical Psychology Review, 31(5), 757-766.

Startup, H., Freeman, D., \& Garety, P. A. (2007). Persecutory delusions and catastrophic worry in psychosis: developing the understanding of delusion distress and persistence. Behaviour research and therapy, 45, 523-537.

Zahra, D., \& Hedge, C. (2010). The reliable change index: Why isn't it more popular in academic psychology. British Psychological Society Psychology Postgraduate Affairs Group Quarterly, 76, 14-19. 
WORRY GROUP FOR PSYCHOSIS

Appendix 1: Feedback Form

Evaluation of Worry Group

You do not need to give your name on this form

1) Overall, how helpful did you find the worry group? (Please circle)

$\begin{array}{lllllll}0 & 1 & 2 & 3 & 4 & 5\end{array}$

$(0=$ not at all helpful $\quad 6=$ extremely helpful $)$

2) Overall, how easy was it to follow what was being discussed? (Please circle)

$\begin{array}{llllllll}0 & 1 & 2 & 3 & 4 & 5\end{array}$

$(0=$ not at all helpful $\quad 6=$ extremely helpful $)$

3) What was the most helpful part about the group?

4) What could we improve about the group?

5) How did you find talking about worry and paranoia in a group with other people?

6) Is there anything else you want to say?

Thank you for taking the time to complete this form 


\section{Figure 1}

Flow diagram of participation in group

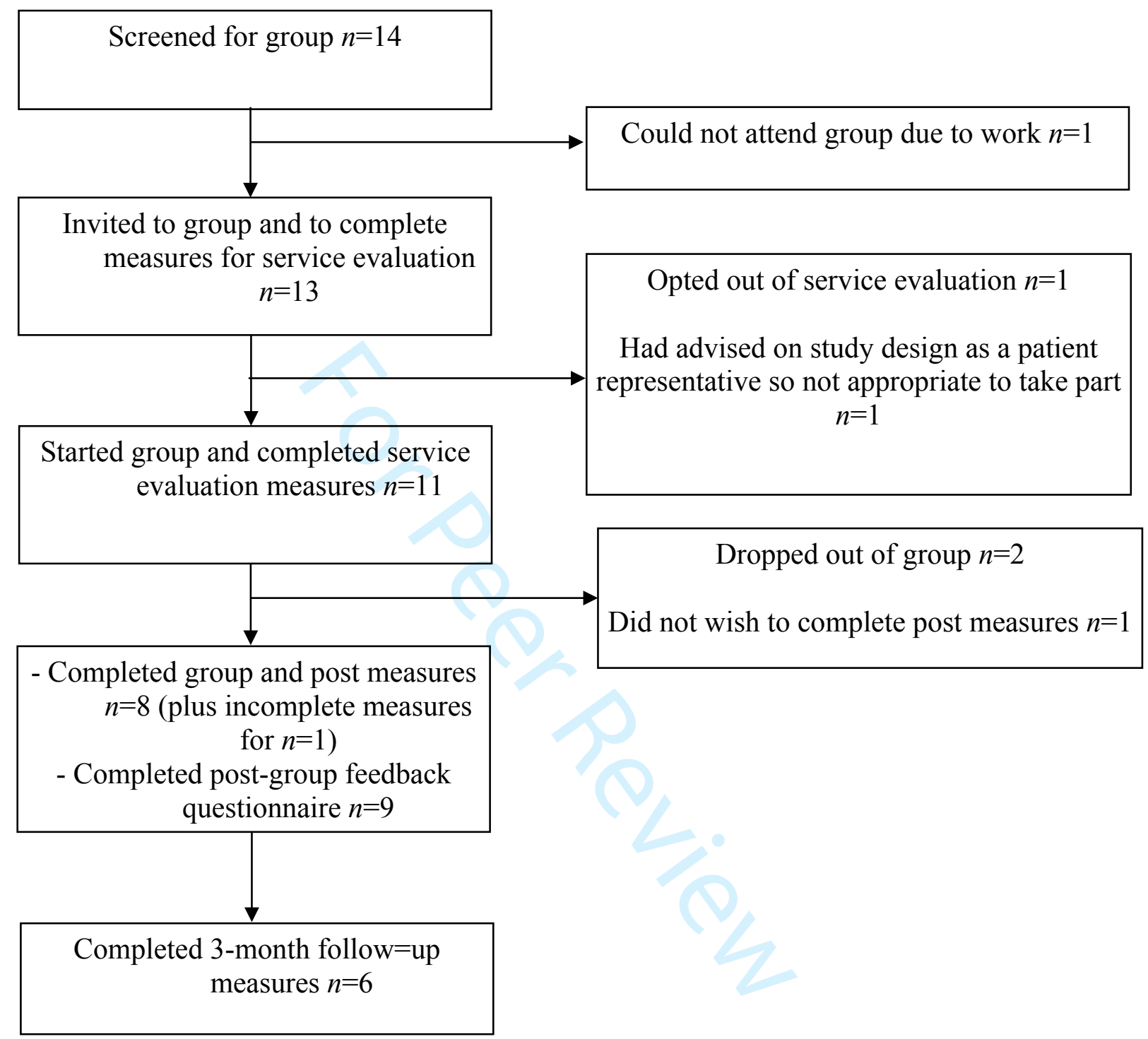


Figure 2

Reliable Change Index results post and follow-up for completers

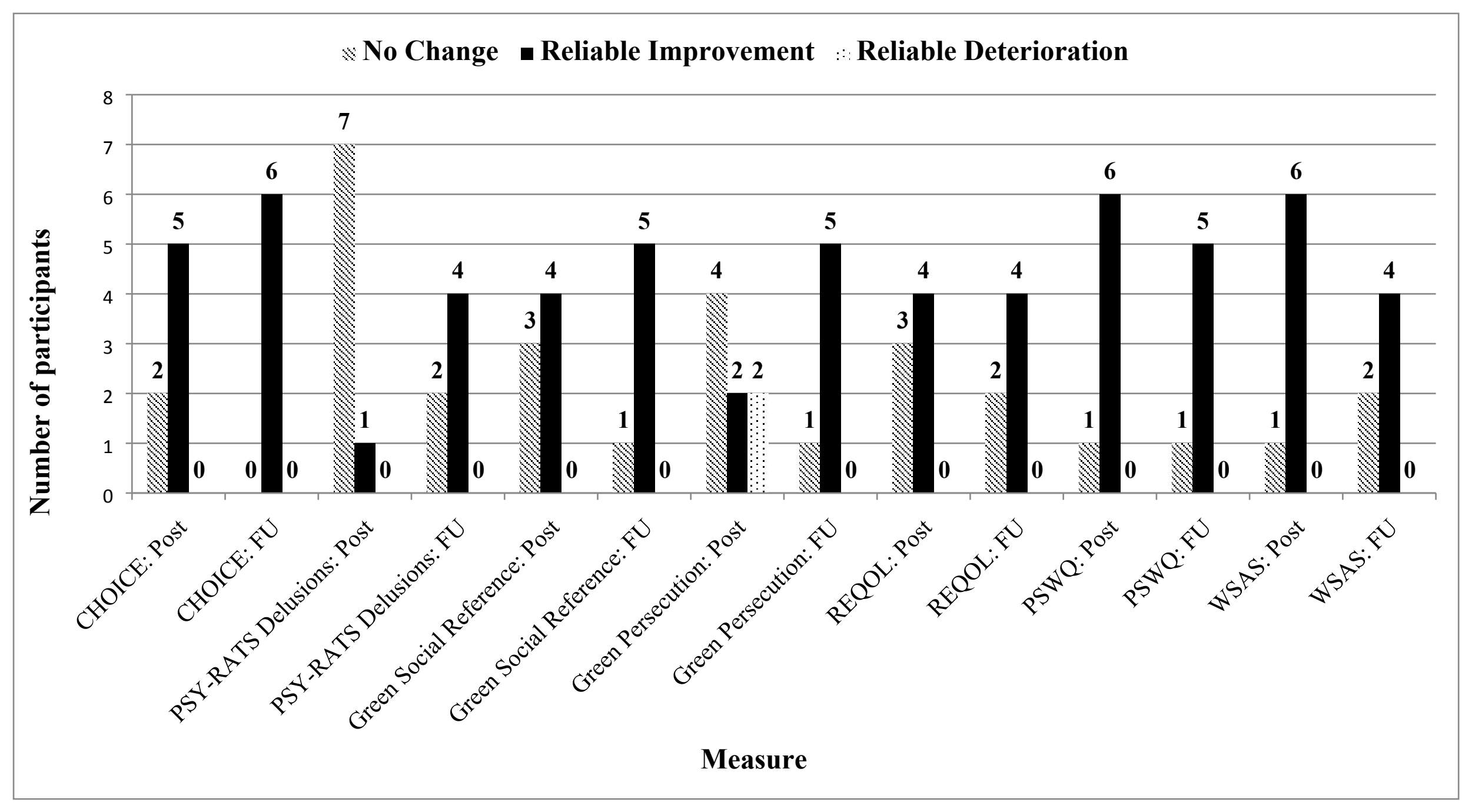


Figure 3

Quantitative post-group feedback

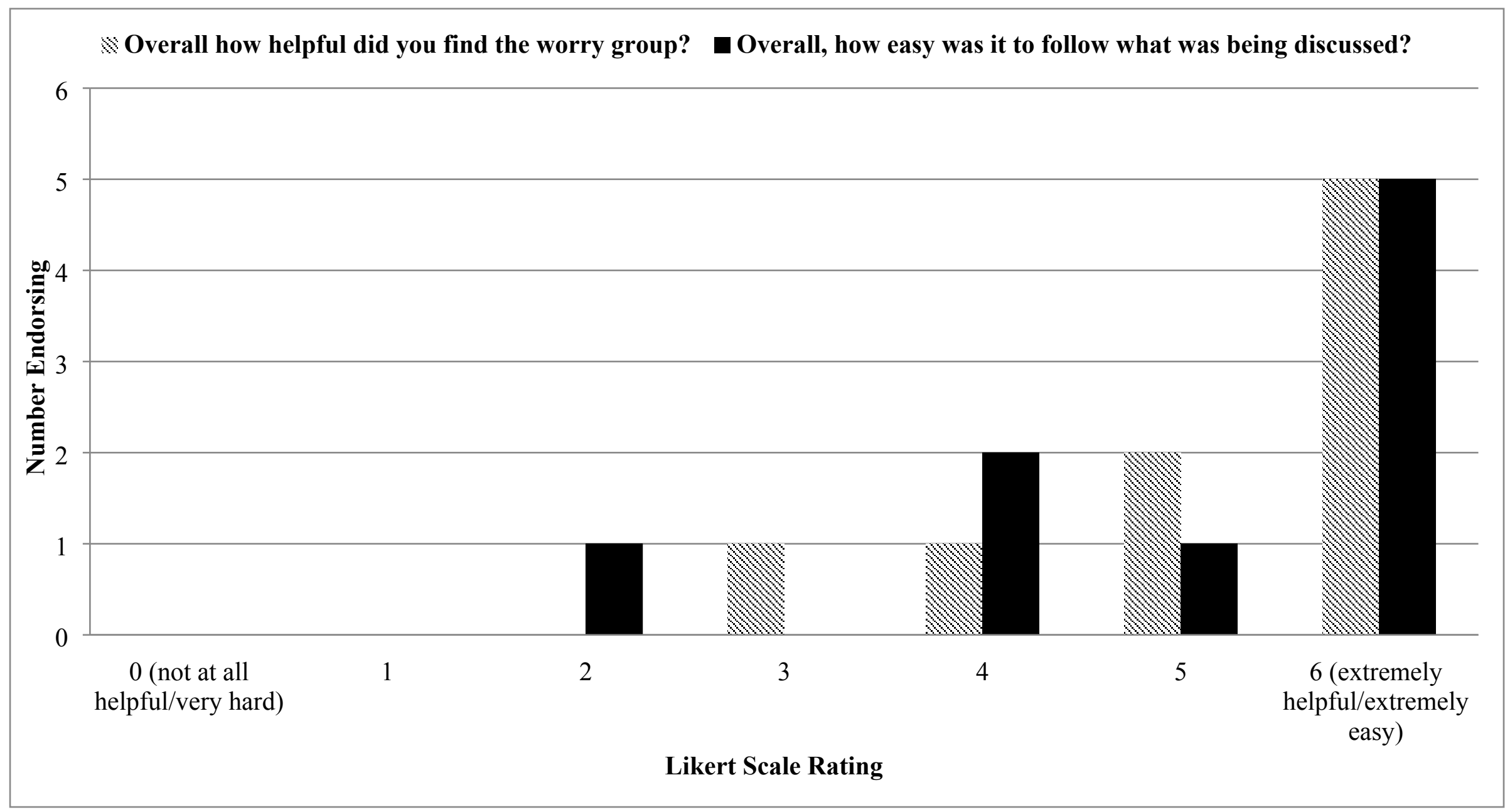




\section{Table 1}

Qualitative post-group feedback (exact quotes from the 9 participants, each bullet points represents a different participant)

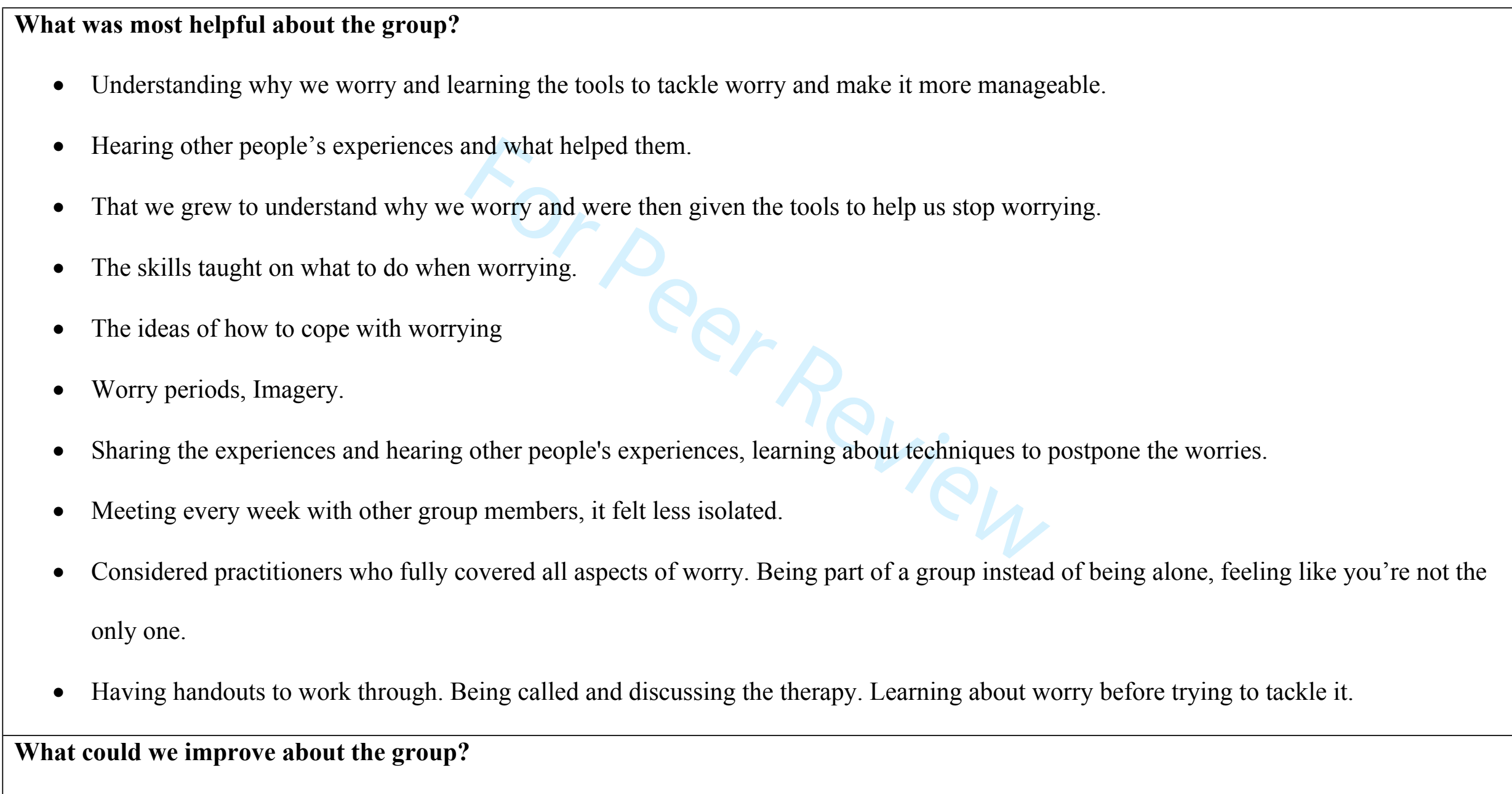


- More people be present.

- Only thing it would have been nice if more people were involved.

- More attendance from other people.

- Perhaps a longer course and a shorter, more condensed session would be helpful.

- The information seemed a bit robortic(sic) and I often found my mind wondering off maybe add some fire into the group.

- Nothing, everything was perfect.

- Makiung(sic) the questions easier to understand.

- To have more time to look at different aspects of (illegible) in detail. Having more people in the group.

\section{How did you find talking about worry and paranoia in a group with other people?}

- I was made to feel at east(sic) with people and found that I wasn't so anxious and that at times it was relatively easy.

- Difficult at first session than not so bad!

- I was made to feel at lease therefore found talking about the above relatively easy!

- Nerve wracking to start, but easier as the weeks went on and we got to know the group.

- Fine, wasn't to bothered by it.

- Difficult. 
- Comfortable.

- OK, I held back on personal stuff.

- I found it easier than I thought I would. Felt completed safe in the group and not judged.

Is there anything else you want to say?

- Thankyou for organising it!

- I am grateful to have been given the chance to do this and course and feel that I have benefitted emensely(sic).

- Being around new people makes me worry and you're attptemed(sic) to help me deal wit this is by putting me in a room with 9 people I have never met before.

- Very helpful.

- The group was very helpful. Thanks.

- Thankyou for the help I received by being in the group.

- A huge thankyou all I have learnt has been a massive change for me. It has opened my eyes to worry. 